\title{
ESTUDO ANATÔMICO DO LENHO DE EUGENIA HIEMALIS CAMBESS. (MYRTACEAE) ${ }^{1}$
}

\section{SIDINEI RODRIGUES DOS SANTOS² JOSÉ NEWTON CARDOSO MARCHIORI ${ }^{3}$}

\section{RESUMO}

É presentemente descrita e ilustrada a madeira de Eugenia hiemalis Cambess., com base em material coletado no Rio Grande do Sul. Foram observadas características anatômicas comuns em Myrtaceae e gênero Eugenia: porosidade difusa; vasos exclusivamente solitários; placas de perfuração simples; pontoações intervasculares alternas e ornamentadas; raios heterogêneos estreitos; fibras com pontoações areoladas; traqueídeos vasicêntricos e cristais. Salientam-se, para a separação da espécie, a presença de parênquima apotraqueal em faixas estreitas e descontínuas, bem como a freqüência e diâmetro poros.

Palavras-chave: Eugenia hiemalis, anatomia da madeira, Myrtaceae.

\section{ABSTRACT}

[Wood anatomy of Eugenia hiemalis Cambess. (Myrtaceae)].

The wood anatomy of Eugenia hiemalis is described, based on samples from Rio Grande do Sul state, Brazil. The anatomical structure includes several characteristics that are common within Myrtaceae and genus Eugenia: exclusively solitary pores; simple perforation plates; alternate and vestured intervessel pits; narrow and heterogeneous rays; fibers with bordered pits; vasicentric tracheids; and crystals. Compared to other native species, the apotracheal parenchyma in narrow discontinous bands, as well as the frequency and diameter of pores, are of special importance to wood identification.

Key words: Eugenia hiemalis, wood anatomy, Myrtaceae.

\section{INTRODUÇÃO}

Pertencente à família Myrtaceae, o gênero Eugenia inclui aproximadamente 1000 espécies de árvores e arbustos, 350 das quais são nativas no Brasil (Landrum \& Kawasaki, 1997) e 31 encontram-se naturalmente no Rio Grande do Sul (Sobral, 2003). Trata-se, sem dúvida, de um dos gêneros mais importantes, tanto em diversidade como em abundância de espécies lenhosas, nas distintas formações vegetacionais do Estado (Klein, 1984).

1 Recebido para publicação em 05-5-2011 e aceito para publicação em 10-6-2011.

2 Biólogo. Bolsista (CNPq - Brasil), doutorando do Programa de Pós-Graduação em Engenharia Florestal, Departamento de Ciências Florestais, Universidade Federal de Santa Maria. CEP 97105-900. Santa Maria, RS, Brasil. sthurt.bio@gmail.com

3 Engenheiro Florestal, Dr. Bolsista de Produtividade em Pesquisa (CNPq - Brasil). Professor Titular do Departamento de Ciências Florestais, Universidade Federal de Santa Maria. Santa Maria, RS, Brasil.
As espécies do gênero prestam-se a diversos usos, especialmente como plantas frutíferas e madeireiras (Record \& Hess, 1949). Como exemplos, citam-se Eugenia uniflora e Eugenia involucrata, os dois representantes sul-riograndenses mais conhecidos do grupo. O primeiro deles é importante frutífera (Oliveira, 2007) e o segundo, além de bons frutos, produz madeira indicada para cabos de ferramentas e construção civil (Marchiori \& Sobral, 1997). Vale acrescentar, ainda, que ambas as espécies são também cultivadas como ornamentais.

Ao contrário das espécies acima comentadas, a maioria das Eugenias nativas é pouco conhecida e investigada. Do ponto de vista anatômico, por exemplo, não ultrapassa a 30 o número de espécies descritas até o momento, número que não corresponde à importância e abrangência do grupo taxonômico em questão.

O presente estudo visa a investigar a estrutura anatômica de Eugenia hiemalis, com vistas a contribuir para o melhor conhecimento e valorização das madeiras nativas do Estado. 
Eugenia hiemalis é arbusto ou árvore pequena (1-3 m), de casca rugosa e folhas inteiras, glabras, discolores, elípticas, elíptico-obovadas ou elíptico-ovadas (35-70 x 18-35 mm), com flores reunidas em fascículos ramifloros ou axilares de até 6 unidades e pedicelos relativamente longos (5-10 mm). Conhecido, popularmente, por guamirim-burro, a espécie distribui-se de Minas Gerais ao Rio Grande do Sul, ocorrendo, neste Estado, em todas as formações vegetacionais (Sobral, 2003).

\section{REVISÃO DE LITERATURA}

Tendo em vista o grande número de espécies e a importância do gênero Eugenia nas floras sul-rio-grandense e brasileira, são notavelmente escassas as referências sobre a estrutura anatômica, disponíveis na literatura.

No Rio Grande do Sul, foram anatomicamente descritas, até o momento, apenas sete espécies: Eugenia involucrata (Marchiori, 1984); Eugenia mansoi (Marchiori \& Santos, 2009); Eugenia uniflora (Santos \& Marchiori, 2009); Eugenia rostrifolia e Eugenia uruguayensis (Marchiori \& Santos, 2010); Eugenia burkartiana (Santos \& Marchiori, 2010a); e Eugenia schuechiana (Santos \& Marchiori, 2011).

Em estudo anatômico, Détienne \& Jacquet (1983) investigaram madeiras de algumas espécies de Eugenia e de outros 7 gêneros da Amazônia brasileira, com vistas à identificação. Mainieri \& Chimelo (1989), por sua vez, descreveram uma espécie não identificada do gênero em questão.

Soffiatti \& Angyalossy-Alfonso (1999) realizaram um estudo comparativo do lenho e casca de Eugenia cerasiflora e Eugenia uniflora, coletadas na Mata Atlântica de São Paulo. Barros et al. (2001; 2003) descreveram cinco espécies de Eugenia, incluindo Eugenia hiemalis, entre outras Myrtaceae nativas no Rio de Janeiro. Marques et al. (2007) investigaram a anatomia da madeira de três espécies do mesmo grupo, com diferentes disponibilidade hídrica, provenientes de remanescentes florestais do Rio de
Janeiro.

Para a espécie em estudo, Barros et al. (2003) referiram, entre outros aspectos: porosidade difusa; poros solitários $(52 \mu \mathrm{m})$; elementos vasculares médios $(708 \mu \mathrm{m})$, com placas de perfuração simples; traqueídeos vasicêntricos; parênquima apotraqueal difuso, difuso-em-agregados e em linhas onduladas com 1 célula de largura; raios heterocelulares, uni até trisseriados; e fibrotraqueídeos de comprimento médio $(1332 \mu \mathrm{m})$. Cabe salientar, todavia, que a ocorrência de espessamentos espiralados e cristais não foi registrada no material analisado.

Acerca da anatomia de Mirtáceas, cabem destacar-se, ainda, os trabalhos clássicos de Record \& Hess (1949) e Metcalfe \& Chalk (1972).

\section{MATERIAL E MÉTODOS}

O material estudado consiste em uma amostra de madeira e respectivo material botânico, coletados em mata ciliar no município de Restinga Seca (Rio Grande do Sul), incorporados à Xiloteca e Herbário do Departamento de Ciências Florestais (HDCF) da Universidade Federal de Santa Maria.

Para a confecção das lâminas histológicas foram extraídos três corpos de prova $(1 \times 2 \times 3 \mathrm{~cm})$ da parte mais externa do lenho, próxima ao câmbio, orientados para obtenção de cortes nos planos transversal, longitudinal radial e longitudinal tangencial. Outro bloquinho foi também retirado, com vistas à maceração.

A confecção das lâminas histológicas seguiu a metodologia descrita em Burger \& Richter (1991). No preparo de lâminas de macerado, seguiu-se o método de Franklin, modificado (Kraus \& Arduin, 1997). Os cortes anatômicos foram tingidos com safra-blau e o macerado apenas com safranina (1\%). Na montagem das lâminas permanentes usou-se Entellan.

A descrição baseou-se nas recomendações do IAWA Committee (1989). No caso da percentagem dos tecidos, foram realizadas 600 determinações ao acaso, com auxílio de contador de laboratório, conforme proposto por Marchiori 
(1980). A frequiência de poros foi obtida de forma indireta, a partir de um quadrado de área conhecida superposto a fotomicrografias de seções transversais da madeira.

As medições foram realizadas em microscópio Carl Zeiss, no Laboratório de Anatomia da Madeira da Universidade Federal de Santa Maria. $\mathrm{Na}$ citação das características quantitativas, os números entre parênteses correspondem aos valores mínimos e máximos observados; o valor que acompanha a média é o desvio padrão. As fotomicrografias foram tomadas em microscópio Olympus CX40, equipado com câmera digital Olympus Camedia C3000, no Laboratório de Anatomia da Madeira da Universidade Federal do Paraná, a quem os autores agradecem.

\section{DESCRIÇÃO ANATÔMICA}

Anéis de crescimento: distintos, delimitados por fina camada de fibras radialmente estreitas e, por vezes, pela presença de delgadas zonas fibrosas no lenho tardio. Porosidade difusa (Figura 1A,B).

Vasos: numerosos $(26 \pm 2,1(24-29)$ poros/ $\mathrm{mm}^{2}$ ), ocupando $11 \pm 5 \%$ do volume da madeira. Poros exclusivamente solitários, circulares ou ovais, pequenos $(61 \pm 11,7(27-92) \mu \mathrm{m})$, de paredes espessas $(3,6 \pm 0,66(2,5-5) \mu \mathrm{m}) \mathrm{e}$ sem padrão definido de organização (Figura $1 \mathrm{~A}, \mathrm{~B})$. Elementos vasculares de comprimento médio $(448 \pm 96(300-760 \mu \mathrm{m})$, com placas de perfuração simples, oblíquas; apêndices geralmente presentes, em uma, mais comumente em ambas as extremidades. Pontoações intervasculares alternas, circulares, pequenas $(4,8 \pm$ $0,42(4,1-5,1) \mu \mathrm{m})$, com abertura em fenda inclusa, ornamentada. Pontoações raio-vasculares com aréolas distintas, semelhantes às intervasculares, embora menores ( $3,1 \pm 0,16(3,1$ $-3,6) \mu \mathrm{m})$ e restritas às margens de raios. Espessamentos espiralados e conteúdos, ausentes.

Parênquima axial: muito distinto das fibras em corte transversal, representando $19 \pm 2,2 \%$ do volume da madeira; em arranjo apotraqueal difuso, difuso-em-agregados e, principalmente, em faixas estreitas e descontínuas, uni ou parcialmente bisseriadas. Parênquima paratraqueal escasso, infrequente (Figura 1A,B). Séries parenquimáticas com $7-8(2-10)$ células e $462 \pm 82(290-650) \mu \mathrm{m}$ de altura (Figura 1F). Cristais prismáticos grandes, muito abundantes e em séries de até 11 unidades, em câmaras distendidas (Figura 1D).

Raios: muito numerosos $(19 \pm 1,2(17-21)$ raios $/ \mathrm{mm}$ ), com $1-2$, menos comumente 3 células de largura (Figura 1E), ocupando $21 \pm 3,1$ $\%$ do volume da madeira. Raios multisseriados de $281 \pm 120(130-570) \mu \mathrm{m}$ e $4-17$, mais comumente 7 - 12 células de altura; heterocelulares, reúnem células procumbentes, na parte multisseriada, e $1-4$ (8) fileiras marginais de células eretas, quadradas e, menos comumente, células procumbentes mais altas do que as do corpo central (Figura 1C). Margens unisseriadas, geralmente mais longas do que o corpo central (Figura 1E,F). Raios unisseriados, de $184 \pm 85(60-410) \mu \mathrm{m}$ de altura, com $1-7$, mais comumente $2-6$ células. Raios axialmente fusionados, frequentes. Células radiais de paredes disjuntas, presentes. Inclusões minerais, células envolventes, células perfuradas e conteúdos, ausentes.

Fibras: com pontoações areoladas de mais de $3 \mu \mathrm{m}$ de diâmetro e aberturas frequentemente cruzadas, nas faces radiais e tangenciais da parede. Fibras de comprimento médio (1086 \pm $96(870-1270) \mu \mathrm{m})$, com $18 \pm 2,5(14-23)$ $\mu \mathrm{m}$ de largura e paredes finas a espessas $(5,4 \pm$ $0,74(4,4-6,9) \mu \mathrm{m})$ (Figura 1B), representando $49 \pm 3,9 \%$ do volume da madeira. Fibras septadas, fibras gelatinosas e espessamentos espiralados, ausentes. Traqueídeos vasicêntricos, presentes.

Outros caracteres: variantes cambiais, tubos laticíferos e taniníferos, canais intercelulares, máculas medulares, células oleíferas, células mucilaginosas e estratificação, ausentes. 

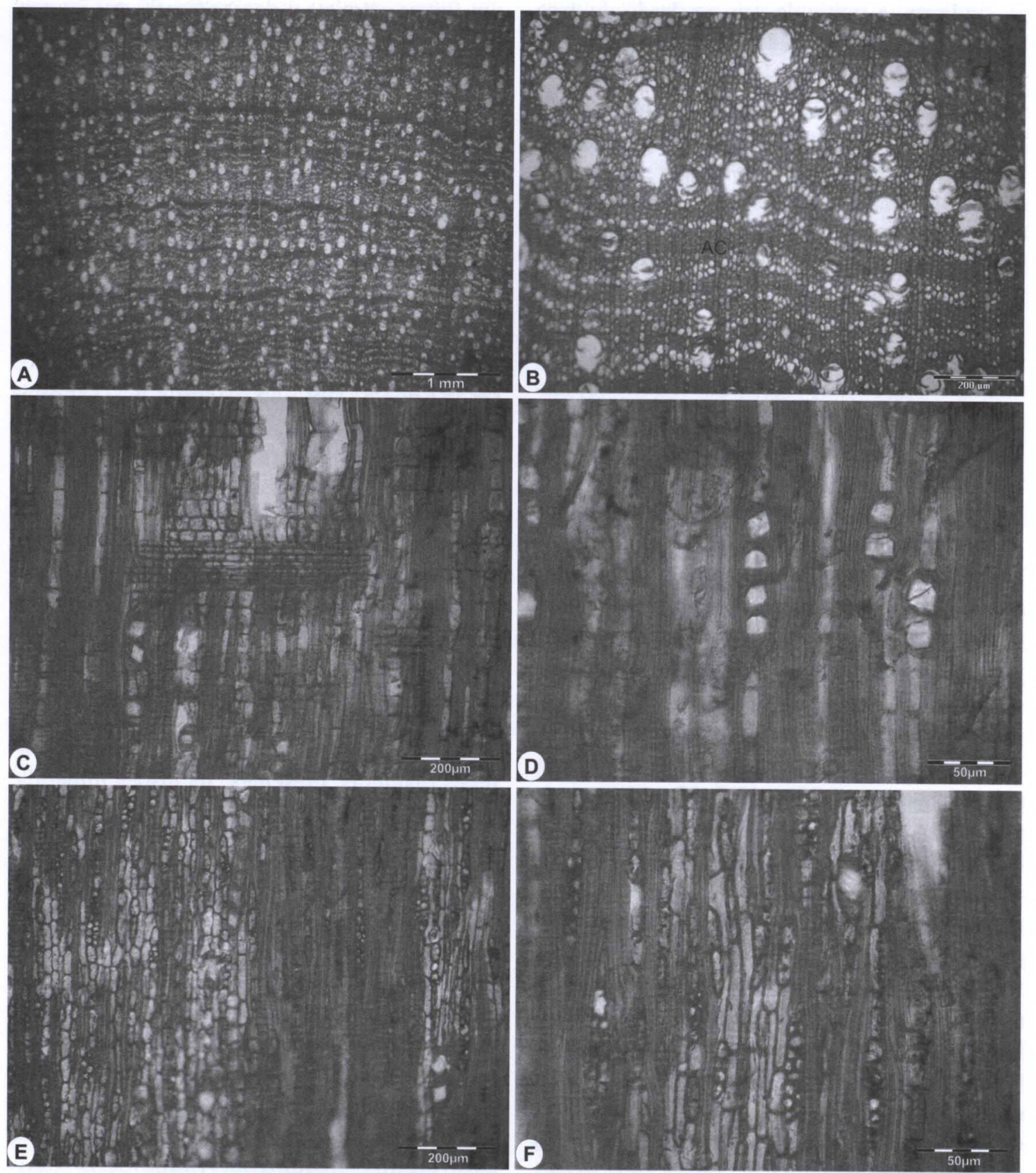

FIGURA 1 - Fotomicrografias da madeira de Eugenia hiemalis. A - Seção transversal, mostrando anel de crescimento distinto (AC), porosidade difusa, poros exclusivamente solitários, parênquima apotraqueal difuso-em-agregados e em faixas estreitas descontínuas (setas). B - Mesma seção, em maior aumento, com destaque para anéis de crescimento (AC), fibras de paredes finas até espessas e o arranjo do parênquima axial. C - Raio heterogêneo, com células procumbentes (no corpo), quadradas e eretas (seção longitudinal radial). D - Parênquima apotraqueal e cristais, em vista radial. E - Aspecto geral da seção longitudinal tangencial. F - Mesma seção, em maior aumento, mostrando raios estreitos, uni e bisseriados e séries de parênquima axial. 
ANÁLISE DA ESTRUTURA ANATÔMICA

As principais características microscópicas da madeira de Eugenia hiemalis correspondem ao descrito para as Myrtaceae, por Record \& Hess (1949), Metcalfe \& Chalk (1972) e Vliet \& Baas (1984), bem como ao referido para as espécies de Eugenia anatomicamente conhecidas. É o caso, entre outros aspectos, da porosidade difusa, dos poros exclusivamente solitários, das placas de perfuração simples, das pontoações intervasculares alternas e ornamentadas, do parênquima apotraqueal, dos raios heterogêneos estreitos, das fibras com pontoações areoladas, dos traqueídeos vasicêntricos e da presença de cristais.

No tocante aos aspectos quantitativos do lenho, os valores observados ficam dentro dos limites citados na literatura para a família e gênero. Cabe salientar, todavia, que os valores de frequência e diâmetro de poros são menores e maiores, respectivamente, do que o referido na literatura para espécies sul-rio-grandenses do grupo.

Da mesma forma, os principais aspectos da anatomia de Eugenia hiemalis correspondem ao descrito para a espécie, por Barros et al (2003), com exceção da presença de cristais e de algumas características quantitativas, notadamente o comprimento de vasos $(708 \mu \mathrm{m})$ e fibras $(1332$ $\mu \mathrm{m})$, que são maiores do que o presentemente encontrado (448 e $1086 \mu \mathrm{m}$, respectivamente).

As diferenças quantitativas podem ser explicadas pela latitude (Santos \& Marchiori, 2010b). A ausência de cristais também não surpreende, uma vez que se trata de aspecto anatômico vinculado a condições ambientais, notadamente à composição do solo e disponibilidade hídrica (Metcalfe \& Chalk,1983; Souza, 2000; Wheeler et al., 2007). Por outro lado, vale lembrar que, à semelhança da espécie em estudo, todos os demais integrantes sul-riograndenses e brasileiros de Eugenia, referidos na literatura, apresentam cristais na madeira.

Apesar da natureza ocasional e da suscetibilidade aos fatores ambientais, a presença ou não de cristais tem sido utilizada, com frequiência, na separação de espécies de Myrtaceae (Dias-Leme et al., 1995; Soffiatti \& AngyalossyAlfonso, 1999). Em Eugenia hiemalis, no entanto, devido à natureza ocasional do caráter, prefere-se não recomendar o uso do mesmo para fins taxonômicos.

Em Eugenia hiemalis, o arranjo do parênquima axial, organizado em faixas ou linhas estreitas e descontínuas, é o aspecto mais marcante na estrutura da madeira. A presença de parênquima em faixas é caráter freqüente em Eugenia, inclusive em espécies sul-riograndenses (Eugenia uniflora, Eugenia rostrifolia, Eugenia schuechiana); o tipo observado (faixas estreitas e descontínuas), no entanto, é pouco comum, verificando-se apenas em Eugenia mansoi, motivo pelo qual mostrase útil na identificação.

Cabe salientar, por fim, que nenhuma característica observada é peculiar a Eugenia hiemalis. $\mathrm{O}$ grande número de caracteres compartilhados com outras espécies de Eugenia e outros gêneros de Myrtoideae (ou tribo Myrteae), dificultam, certamente - mas não impedem -, a identificação do material em estudo.

\section{REFERÊNCIAS BIBLIOGRÁFICAS}

BARROS, C.F.; CALLADO, C.H.; MARCON, M.L.; COSTA, C.G.; CUNHA, M.; LIMA, H.R.P.; MARQUETE, O. Madeiras da Mata Atlântica. Anatomia do lenho de espécies ocorrentes nos remanescentes florestais do estado do Rio de Janeiro, Brasil. Rio de Janeiro: Instituto de Pesquisas Jardim Botânico do Rio de Janeiro, 2001. $94 \mathrm{p}$.

BARROS, C.F.; CALLADO, C.H.; CUNHA, M.; MARCON, M.L.F.; TAMAIO, N.; MARQUETE, O.; COSTA, C.G. Madeiras da Mata Atlântica. Anatomia do lenho de espécies ocorrentes nos remanescentes florestais do estado do Rio de Janeiro, Brasil. Rio de Janeiro: Instituto de Pesquisas Jardim Botânico do Rio de Janeiro, 2003. 86 p. 
BURGER, L.M.; RICHTER, H.G. Anatomia da Madeira. São Paulo: Ed. Nobel, 1991. 154 p.

DÉTIENNE, P.; JACQUET, P. Atlas d'identification des bois de l'Amazonie et des regions voisines. Nogent-sur-Marne: Centre Technique Forestier Tropical, 1983.

DIAS-LEME, C.L.; GASSON, P.; LUGHADA, E.N. Wood anatomy of four Myrtaceae genera in the subtribe Myciinae from South America. IAWA Bulletin, n. 16, p. 87-95, 1995.

IAWA COMMITTEE. IAWA list of microscopic features for hardwood identification. IAWA Bulletin, v.10, n. 3, p.218-359, 1989.

KRAUS, J.E.; ARDUIN, M. Manual básico de métodos em Morfologia Vegetal. Rio de Janeiro: EDUR, 1997. 198 p.

KLEIN, R.M. Importância sociológica das Mirtáceas nas florestas rio-grandenses. Anais do XXXIV Congresso Nacional de Botânica (Porto Alegre), p. $367-375,1984$.

LANDRUM, L.R.; KAWASAKI, M.L. The genera of Myrtaceae in Brasil: an illustrated synoptic treatment and identification keys. Brittonia, n. 49, p. 508-536, 1997.

MAINIERI, C.; CHIMELO, J.P. Fichas de características das madeiras brasileiras. São Paulo: Instituto de Pesquisas Tecnológicas, Divisão de Madeiras, 1989. 418p.

MARQUES, P.A.; ARAUJO, G.U.C.; BARROS, C.F.; CALLADO, C.H. Anatomia do lenho de três espécies de Eugenia L. (Myrtaceae) de mata e restinga. Revista Brasileira de Biociências, v. 5, p. 801-803, 2007.

MARCHIORI, J.N.C. Comprovação da viabilidade da utilização da secção longitudinal tangencial para a determinação histométrica dos elementos axiais do xilema secundário. In: Anais do IV Congresso Florestal Estadual, Nova Prata, RS, p. 180-184, 1980.

MARCHIORI, J.N.C. Anatomia da madeira de Eugenia involucrata DC. (Myrtaceae). Ciência e Natura, Santa Maria, v. 6, p. 127-136, 1984.

MARCHIORI, J.N.C.; SANTOS, S.R. Anatomia do xilema secundário de Eugenia mansoi $\mathrm{O}$. Berg (Myrtaceae). Balduinia, Santa Maria, n. 16, p. 6-12, 2009.

MARCHIORI, J.N.C.; SANTOS, S.R. Anatomia da madeira de duas espécies de Eugenia L. (Myrtaceae). Balduinia, Santa Maria, n. 21, p. 15-21, 2010.

MARCHIORI, J.N.C.; SOBRAL, M. Dendrologia das Angiospermas: Myrtales. Santa Maria: Ed. UFSM, 1997. 304 p.

METCALFE, C.R.; CHALK, L. Anatomy of the Dicotyledons. Wood structure and conclusions of the general introdution. Oxford: Clarendon Press, 1983.

METCALFE, C.R.; CHALK, L. Anatomy of the Dicotyledons. Oxford: Clarendon Press, 1972. 1500p.

OLIVEIRA, F. Aspectos da vegetação arbórea encontrada na orla da Praia da Alegria no município de Guaíba, RS, Brasil. Caderno de Pesqui$s a$, série Biológica, Universidade de Santa Cruz do Sul, v. 19, p. 6-17, 2007.

RECORD, S.J.; HESS R.W. Timbers of the New World. New Haven: Yale University Press, 1949. 640p.

SANTOS, S.R.; MARCHIORI, J.N.C. Anatomia da madeira de Eugenia uniflora L. (Myrtaceae). Balduinia, Santa Maria, n. 17, p. 11-16, 2009.

SANTOS, S.R.; MARCHIORI, J.N.C. Tendências anatômicas na flora sul-rio-grandense. 1 - Elementos vasculares. Balduinia, Santa Maria, n. 21, p. 1-14, 2010 b.

SANTOS, S.R.; MARCHIORI, J.N.C. Anatomia das madeiras de Eugenia burkartiana (D. Legrand) D. Legrand e Myrciaria cuspidata O. Berg, duas Myrtoídeas nativas no Rio Grande do Sul. Balduinia, Santa Maria, n. 22, p. 15-22, 2010a.

SANTOS, S.R.; MARCHIORI, J.N.C. Estudo anatômico do lenho de Eugenia schuechiana O. Berg. Balduinia, Santa Maria, n. 26, p. 27-32, 2011.

SOBRAL, M. A família Myrtaceae no Rio Grande do Sul. São Leopoldo: Unisinos, 2003. 215p.

SOFFIATTI, P.; ANGYALOSSY-ALFONSO, V. Estudo anatômico comparativo do lenho e da casca de duas espécies de Eugenia L. (Myrtaceae). Revista Brasileira de Botânica, São Paulo, v. 22, n. 2, p. 175-184, 1999.

SOUZA, L.P. Anatomia ecológica do lenho de Psidium cattleyanum Sabine (Myrtaceae) e sua relação com a morfologia foliar e a cor dos frutos. 2000. 76f. Dissertação (Mestrado em Botânica) - Universidade Federal do Paraná, Curitiba, 2000.

VLIET, G.J.C. van; BAAS, P. Wood anatomy and classification of the Myrtales. Annals of the Missouri Botanical Garden, n. 71, p. 783-800, 1984.

WHEELER, E.A; BAAS, P.; RODGERS, S. Variations in Dicot wood anatomy: a global analysis based on the insidewood database. IAWA Journal, v. 28, n. 3, p. 229-258, 2007. 